\title{
Rotation-Torsion Interaction in the Ground State Microwave Spectrum of Trans-gauche Ethylnitrite
}

\author{
Ch. Keussen and H. Dreizler \\ Abteilung Chemische Physik im Institut für Physikalische Chemie der Universität Kiel \\ Z. Naturforsch. 46a, 590-594 (1991); received March 30, 1991

\begin{abstract}
We investigated a small rotation-torsion interaction in the microwave spectrum of trans-gauche
\end{abstract} \\ ethylnitrite, $\mathrm{CH}_{3} \mathrm{CH}_{2} \mathrm{ONO}$. We found the difference between the two lowest energy levels of the \\ torsion around the $\mathrm{C}-\mathrm{O}$ bond axis to be $24.8(8) \mathrm{kHz}$. The high resolution of microwave Fourier \\ transform (MWFT) spectroscopy was necessary to measure the resulting small splittings.
}

\section{Introduction}

Several investigations were made on the microwave spectrum of ethylnitrite in recent years. Turner [1] assigned the spectra of three conformers called cistrans, cis-gauche, and trans-gauche ethylnitrite. They are shown in Figure 1. Endo et al. [2] recorded the spectra of some istopomers and determined partial structures. In 1988 we published the ground state quadrupole coupling constants of all three conformers [3] determined by microwave Fourier transform (MWFT) spectroscopy. We found an additional splitting of the c-type transitions of trans-gauche ethylnitrite. Turner [1] found a similar, but much larger splitting of the $c$-type transitions of the named conformer in an excited state of the torsion around the $\mathrm{C}-\mathrm{O}$ bond axis. This splitting is caused by the interaction between the overall rotation and the tunneling motion through the barrier separating the two equivalent gauche-conformations. The splitting in the ground torsional state should have the same reason. To confirm this hypothesis, further research had to be done.

\section{Experimental}

Ethylnitrite was purchased from Fa. MerckSchuchardt, Darmstadt, in a purity of $85 \%$ and distilled under low pressure for further purification. The measurements were made with waveguide MWFT spectrometers in the frequency range from 3.8 to

Reprint requests to Prof. Dr. H. Dreizler, Institut für Physikalische Chemie der Universität Kiel, Ohlshausenstr. 40, W-2300 Kiel 1, Germany.
$40 \mathrm{GHz}[4-8]$ at pressures between 0.025 and $0.2 \mathrm{~Pa}$ and temperatures between -30 and $-55^{\circ} \mathrm{C}$. We focused our attention on recording additional $c$-type transitions to gain more information on the rotationtorsion interaction.

The Fourier transformation of a transient emission signal into the frequency domain causes line shape deformations, especially in the case of narrow multipletts [9]. To eliminate these overlap effects, the frequencies of the transitions were determined by a least squares fit of the time domain signal [10]. Because this fit program was improved since our last publication on ethylnitrite [3], all transitions given there were refitted to gain more accurate hyperfine frequencies. We focused our attention on measuring additional $c$-type transitions with their torsional hyperfine structure. A total of $5 a$ - and $29 c$-type transitions was measured. A list of all transitions is given in Table 1. For an example see Fig. 3 of [3]. To calculate the hypothetical center frequencies we used the quadrupole coupling
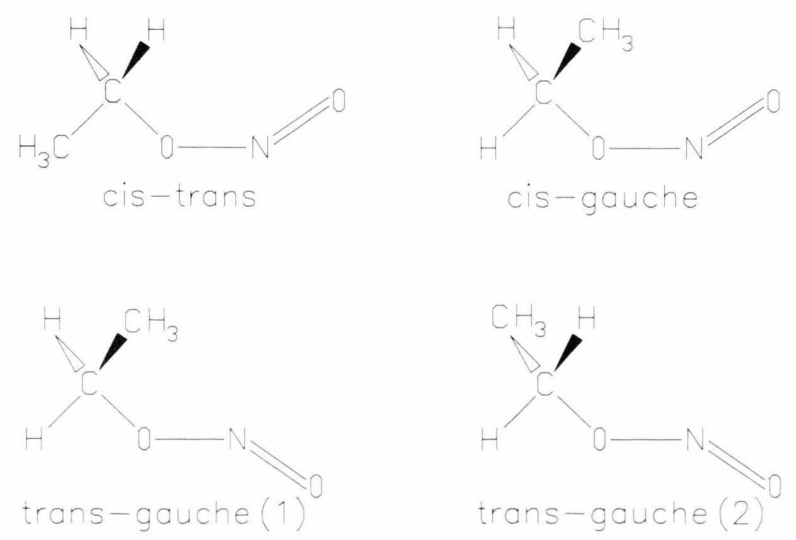

Fig. 1. Rotational isomers of ethylnitrite. 
Ch. Keussen and H. Dreizler - The Ground State Microwave Spectrum of Trans-gauche Ethylnitrite

Table 1. Rotational transitions of trans-gauche ethylnitrite. $v_{\text {obs }}$ : measured frequency [MHz], $v_{\mathrm{c}}$ : hypothetical unsplit line frequency $[\mathrm{MHz}]$ calculated by adding the hfs-splittings to the frequencies of the components, $\delta_{c}$ : deviation of the centrifugal distortion calculation $[\mathrm{MHz}]$.

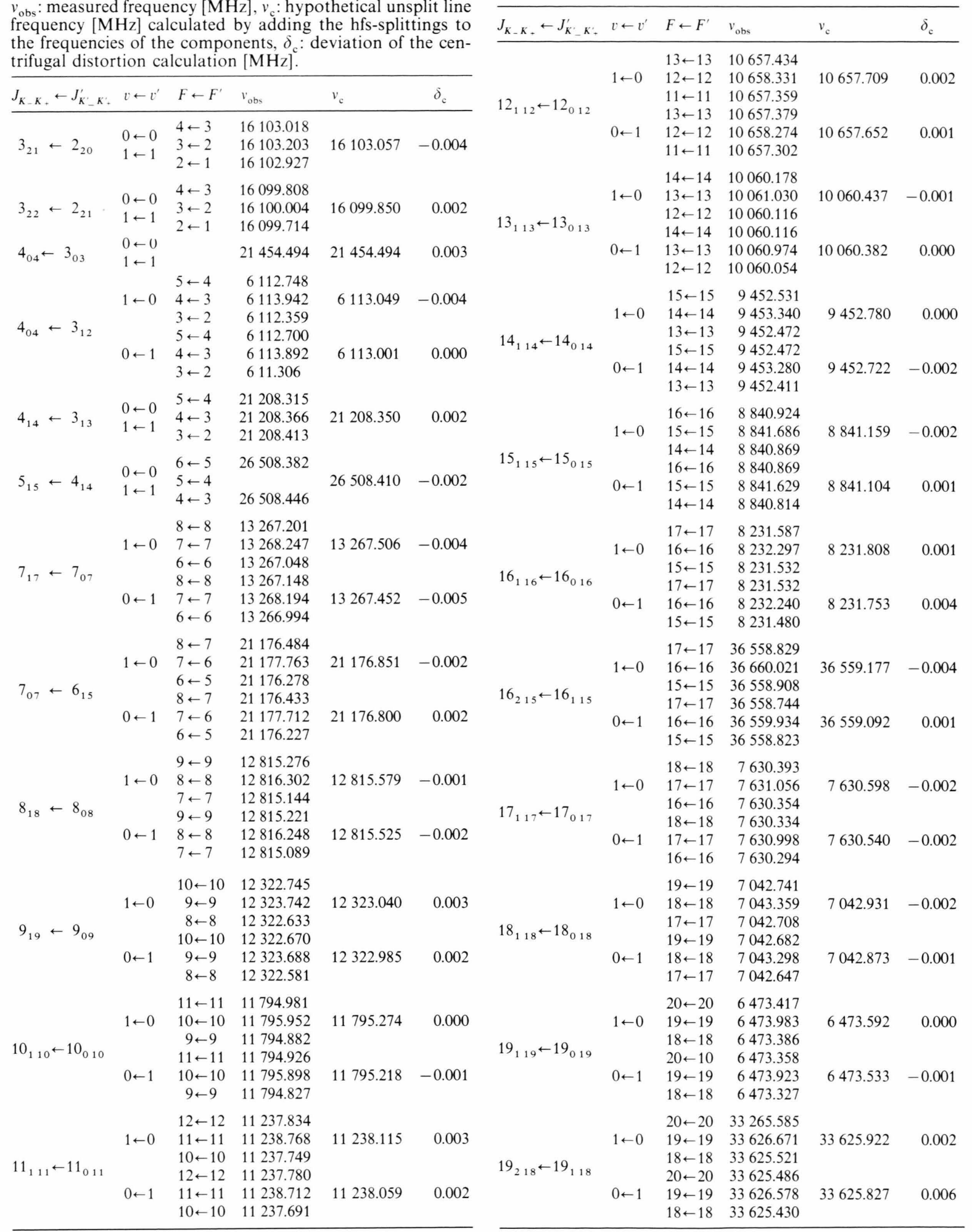

Table 1 (continued) 
Table 1 (continued)

\begin{tabular}{|c|c|c|c|c|c|}
\hline$J_{K_{-} K_{+}} \leftarrow J_{K_{-}^{\prime} K_{-}}^{\prime}$ & $v \leftarrow v^{\prime}$ & $F \leftarrow F^{\prime}$ & $v_{\text {obs }}$ & $v_{\mathrm{c}}$ & $\delta_{\mathrm{c}}$ \\
\hline $20_{120} \leftarrow 20_{020}$ & $1 \leftarrow 0$ & $\begin{array}{l}21 \leftarrow 21 \\
20 \leftarrow 20 \\
19 \leftarrow 19 \\
21 \leftarrow 21 \\
20 \leftarrow 20 \\
19 \leftarrow 19\end{array}$ & 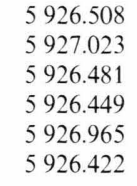 & 5926.608 & 0.002 \\
\hline $20_{219} \leftarrow 20_{119}$ & $1 \leftarrow 0$ & $\begin{array}{l}21 \leftarrow 21 \\
20 \leftarrow 20 \\
19 \leftarrow 19 \\
21 \leftarrow 21 \\
20 \leftarrow 20 \\
19 \leftarrow 19\end{array}$ & $\begin{array}{ll}32 & 538.124 \\
32 & 539.191 \\
32 & 538.071 \\
32 & 538.028 \\
32 & 539.097 \\
32 & 537.970\end{array}$ & 32538.364 & 0.001 \\
\hline $21_{121} \leftarrow 21_{021}$ & $1 \leftarrow 0$ & $\begin{array}{l}22 \leftarrow 22 \\
21 \leftarrow 21 \\
20 \leftarrow 20 \\
22 \leftarrow 22 \\
21 \leftarrow 21 \\
20 \leftarrow 20\end{array}$ & $\begin{array}{l}5405.340 \\
5405.810 \\
5405.317 \\
5405.281 \\
5405.752 \\
5405.259\end{array}$ & 5405.426 & 0.001 \\
\hline $22_{121} \leftarrow 22_{021}$ & $1 \leftarrow 0$ & $\begin{array}{l}23 \leftarrow 23 \\
22 \leftarrow 22 \\
21 \leftarrow 21 \\
23 \leftarrow 23 \\
22 \leftarrow 22 \\
21 \leftarrow 21\end{array}$ & $\begin{array}{l}4912.464 \\
4912.888 \\
4912.444 \\
4912.406 \\
4912.830 \\
4912.386\end{array}$ & 4912.537 & 0.000 \\
\hline $22_{221} \leftarrow 22_{121}$ & $0 \leftarrow 1$ & $\begin{array}{l}23 \leftarrow 23 \\
22 \leftarrow 22 \\
21 \leftarrow 21 \\
23 \leftarrow 23 \\
22 \leftarrow 22 \\
21 \leftarrow 21\end{array}$ & $\begin{array}{l}30269.910 \\
30270.959 \\
30269.862 \\
30269.806 \\
30270.856 \\
30269.753\end{array}$ & 30270.133 & -0.008 \\
\hline $24_{223} \leftarrow 24_{123}$ & $1 \leftarrow 0$ & $\begin{array}{l}25 \leftarrow 25 \\
24 \leftarrow 24 \\
23 \leftarrow 23 \\
25 \leftarrow 25 \\
24 \leftarrow 24 \\
23 \leftarrow 23\end{array}$ & $\begin{array}{l}27904.838 \\
27905.842 \\
27904.769 \\
27904.735 \\
27905.732 \\
27904.692\end{array}$ & 27905.152 & -0.003 \\
\hline $25_{224} \leftarrow 25_{124}$ & $0 \leftarrow 1$ & $\begin{array}{l}26 \leftarrow 26 \\
25 \leftarrow 25 \\
24 \leftarrow 24 \\
26 \leftarrow 26 \\
25 \leftarrow 25 \\
24 \leftarrow 24\end{array}$ & $\begin{array}{l}26697.339 \\
26698.315 \\
26697.296 \\
26697.225 \\
26698.202 \\
26697.183\end{array}$ & 26697.644 & 0.004 \\
\hline$\leftarrow 25_{125}$ & $1 \leftarrow 0$ & $\begin{array}{l}27 \leftarrow 27 \\
26 \leftarrow 26 \\
25 \leftarrow 25 \\
27 \leftarrow 27 \\
26 \leftarrow 26 \\
25 \leftarrow 25\end{array}$ & $\begin{array}{l}25479.733 \\
25480.689 \\
25479.695 \\
25479.619 \\
25480.572 \\
25479.580\end{array}$ & 25480.031 & -0.001 \\
\hline$\leftarrow 27_{126}$ & $1 \leftarrow 0$ & $\begin{array}{l}28 \leftarrow 28 \\
27 \leftarrow 27 \\
26 \leftarrow 26 \\
28 \leftarrow 28 \\
27 \leftarrow 27 \\
26 \leftarrow 26\end{array}$ & $\begin{array}{l}24257.192 \\
24257.156 \\
24258.119 \\
24257.071 \\
24257.035 \\
24257.997\end{array}$ & 24257.481 & -0.001 \\
\hline $28_{22-7} \leftarrow 28_{127}$ & $0 \leftarrow 1$ & $\begin{array}{l}29 \leftarrow 29 \\
28 \leftarrow 28 \\
27 \leftarrow 27 \\
29 \leftarrow 29 \\
28 \leftarrow 28 \\
27 \leftarrow 27\end{array}$ & $\begin{array}{ll}23 & 035.009 \\
23 & 035.903 \\
23 & 034.977 \\
23 & 034.881 \\
23 & 035.779 \\
23 & 034.847\end{array}$ & 23035.288 & -0.001 \\
\hline
\end{tabular}

Table 1 (continued)

\begin{tabular}{llllll}
\hline$J_{K_{-} K_{+}} \leftarrow J_{K_{-}^{\prime} K_{+}}^{\prime} v \leftarrow v^{\prime}$ & $F \leftarrow F^{\prime}$ & $v_{\mathrm{obs}}$ & $v_{\mathrm{c}}$ & $\delta_{\mathrm{c}}$ \\
\hline & & $30 \leftarrow 30$ & 21818.558 & & \\
& $1 \leftarrow 0$ & $29 \leftarrow 29$ & 21819.418 & 21818.824 & -0.002 \\
$29_{228} \leftarrow 29_{128}$ & & $28 \leftarrow 28$ & 21818.525 & & \\
& $0 \leftarrow 1$ & $29 \leftarrow 29$ & 21818.419 & & \\
& & $218 \leftarrow 28$ & 21818.388 & 21818.689 & 0.002 \\
& & $31 \leftarrow 31$ & 20613.203 & & \\
$30_{229} \leftarrow 30_{129}$ & & $30 \leftarrow 30$ & 20614.040 & 20613.460 & -0.002 \\
& $0 \leftarrow 1$ & $31 \leftarrow 31$ & 20613.065 & & \\
& & $30 \leftarrow 30$ & 20613.903 & 20613.322 & 0.002 \\
& $29 \leftarrow 29$ & 20613.034 & & \\
\hline
\end{tabular}

constants from [3]. Since they are determined with sufficient accuracy, no effort was made to improve them further.

\section{Theoretical Background}

To fit the rotation-torsion interaction we assumed the molecule to be rigid with exception of the large amplitude internal torsion around the $\mathrm{C}-\mathrm{O}$ bond axis. In Fig. 2 one can see that this bond axis is nearly parallel to the $a$-principal inertia axis. To simplify the setup of the Hamiltonian we made the approximation that the torsional angular momentum couples with the a-component $P_{a}$ of the total angular momentum only. It is then possible to fit the spectra of the hypothetical center frequencies to a Hamiltonian [1] in the so called reduced axis system. The potential function of the torsion is a double minimum potential shown in Figure 3. The Hamiltonian in a $\mathrm{II}^{r}$-representation $(a=y, b=z, c=x)$ can be written as follows:

$$
\begin{aligned}
& \hat{\boldsymbol{H}}=\left(\begin{array}{ll}
\hat{\boldsymbol{H}}_{00} & \hat{\boldsymbol{H}}_{01} \\
\hat{\boldsymbol{H}}_{01} & \hat{\boldsymbol{H}}_{11}
\end{array}\right), \\
& \hat{\boldsymbol{H}}_{00}=A_{0} \hat{P}_{a}^{2}+B_{0} \hat{P}_{b}^{2}+C_{0} \hat{P}_{c}^{2}+\hat{\boldsymbol{H}}_{c d 00}, \\
& \hat{\boldsymbol{H}}_{11}=A_{1} \hat{P}_{a}^{2}+B_{1} \hat{P}_{b}^{2}+C_{1} \hat{P}_{c}^{2}+\hat{\boldsymbol{H}}_{c d 11}+\Delta E_{01}, \\
& \hat{\boldsymbol{H}}_{01}=F_{01}\left(\hat{P}_{b} \hat{P}_{c}+\hat{P}_{c} \hat{P}_{b}\right) .
\end{aligned}
$$

$A_{0}, B_{0}, C_{0}, A_{1}, B_{1}, C_{1}$ : rotational constants in the torsional states $v=0,1$, respectively,

$\hat{\boldsymbol{H}}_{c d}$ : Hamiltonian of the centrifugal distortion up to sixth order according to Watson's A reduction,

$\Delta E_{0}$ : energy difference of the two torsional states $v=0$ and $v=1$ (see Fig. 3),

$F_{01}$ : fit parameter of the rotation-torsion interaction. 


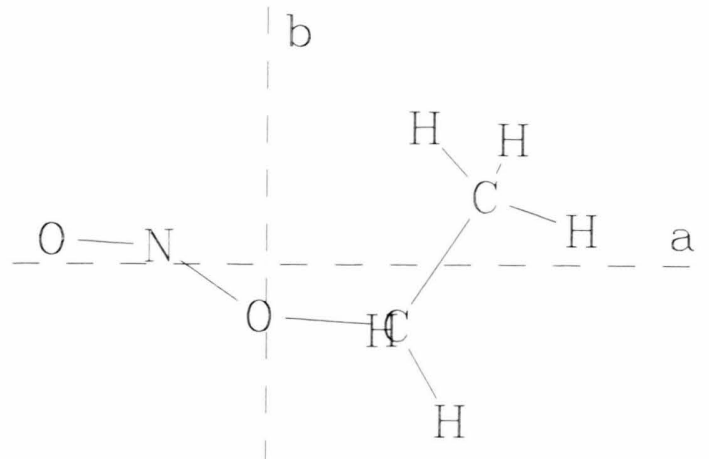

Fig. 2. Trans-gauche ethylnitrite in a projection on the $a, b$ principal inertia axis plane.

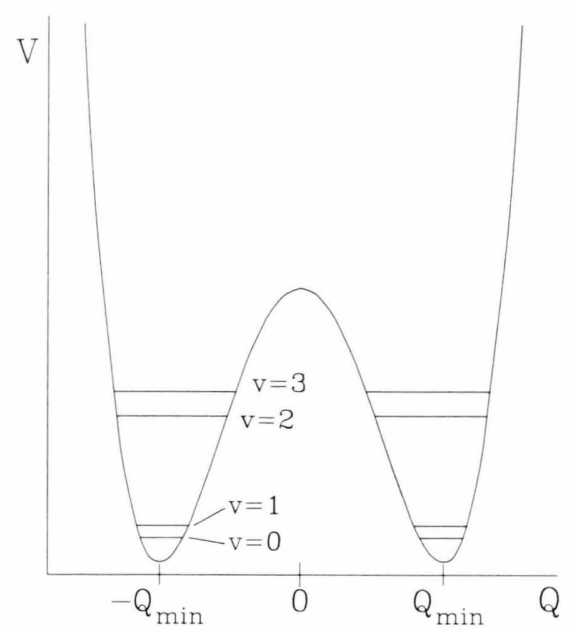

Fig. 3. Potential energy function of the symmetric double minimum potential. Q: torsional coordinate, V: potential energy.

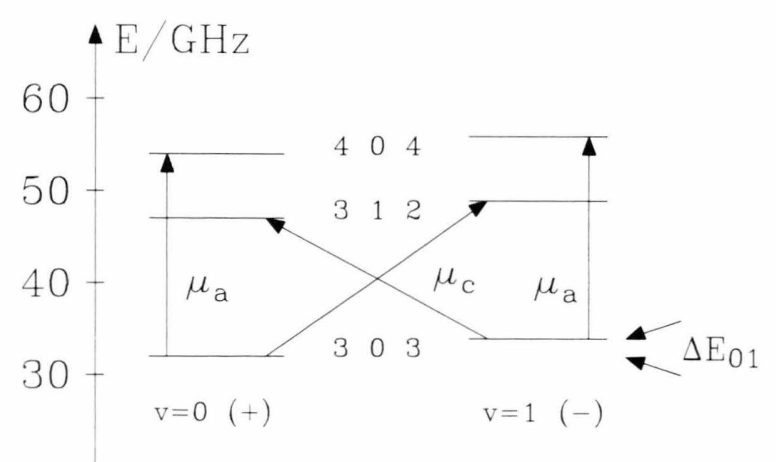

Fig. 4. Some energy levels and possible transitions of transgauche ethylnitrite.
Table 2. Rotational and centrifugal distortion constants written in the notation of sums and differences explained in the text. $A, B, C$ : rotational constants, $\Delta_{J}, \Delta_{J K}, \Delta_{K}, \delta_{J}, \delta_{K}, \Phi_{J}$, $\Phi_{J K}, \Phi_{K J}, \Phi_{K}, \phi_{J}, \phi_{J K}, \phi_{K}$ : fourth and sixth order centrifugal distortion constants according to Watson's A-reduction, rotation-torsion interaction constants $\Delta E_{01}, F_{01}$ : explanation see text, $\varkappa$ : asymmetry parameter, $\sigma$ : standard deviation of the fit, standard errors in brackets, $\Delta \bar{v}_{\text {exp }}$ : mean experimental torsion splitting, largest correlation coefficient: $\left(\left|\Delta_{J}, \Delta_{K}\right|\right)$ $=-0.992$.

\begin{tabular}{|c|c|c|c|}
\hline $\begin{array}{l}A \\
B \\
C \\
\Delta_{J} \\
\Delta_{J K} \\
\Delta_{K} \\
\delta_{J} \\
\delta_{K} \\
\Phi_{J} \\
\Phi_{J K}\end{array}$ & $\begin{array}{rr}17639.500(10) \mathrm{MHz} \\
2745.031(6) \mathrm{MHz} \\
2620.852(5) \mathrm{MHz} \\
628.4 \quad(29) \mathrm{kHz} \\
-1979.2 \quad(80) \mathrm{kHz} \\
1357.0 \quad(48) \mathrm{kHz} \\
-313.7 \quad(15) \mathrm{kHz} \\
673.7 \quad(30) \mathrm{kHz} \\
-92 . & (11) \mathrm{Hz} \\
0.683(15) \mathrm{kHz}\end{array}$ & $\begin{array}{l}\Phi_{K J} \\
\Phi_{K} \\
\phi_{J} \\
\phi_{J K} \\
\phi_{K} \\
\Delta E_{01} \\
F_{01} \\
\Delta C \\
\Delta B \\
\Delta\left(\Delta_{K}\right)\end{array}$ & $\begin{array}{cc}-1.14(30) \mathrm{kHz} \\
0.55(17) \mathrm{kHz} \\
43.8(52) \mathrm{Hz} \\
-279 . \quad(71) \mathrm{Hz} \\
271 . \quad(83) \mathrm{Hz} \\
24.8 \quad(8) \mathrm{kHz} \\
7.2 \quad(18) \mathrm{kHz} \\
12 . \quad(2) \mathrm{Hz} \\
54 . \quad(6) \mathrm{Hz} \\
0.007(9) \mathrm{Hz}\end{array}$ \\
\hline$\sigma:$ & $5 \mathrm{KHZ}$ & $\Delta v_{\exp }$. & 12 \\
\hline
\end{tabular}

We assume that only the first two torsional states $(v=0,1$ in Fig. 3$)$ take part in the rotation-torsion interaction. The Hamiltonian (1 b), (1 c) includes the usual centrifugal distortion $\hat{\boldsymbol{H}}_{c d}$ according to Watson [12]. This implies that the rigidity for vibrational degrees of freedom has been lifted by allowing small amplitude vibrations.

Only a short sketch of the development of this Hamiltonian is outlined in [11]. We therefore performed a careful step by step development of the Hamiltonian and confirmed especially that the transformation from the principal axis system of inertia to the reduced axis system is in fact a contact transformation. That means that the angular momenta components in the reduced axis system obey the normal commutation relations of quantum mechanical angular momenta and can be treated in the usual manner. Details of these rather extended considerations are given in [13].

The $c$-dipole moment $\mu_{c}$ is an odd function of the $\mathrm{C}-\mathrm{O}$ torsional angle, hence $c$-type transitions connect the $v=0$ and $v=1$ torsional states and show a splitting of approximately twice the energy difference $\Delta E_{01}$ (see Figure 4).

The fit of the measured transitions to the Hamiltonian was carried out with a modified version of the program MALON*.

* Original written by L. Halonen, P. H. Turner, and J. Randell. 


\section{Results and Discussion}

From the splittings of the $c$-type transitions (ca. $45-140 \mathrm{kHz}$ ) one expects the torsional energy difference $\Delta E_{01}$ to be in the range between 20 and $70 \mathrm{kHz}$. This small difference entails that the rotational and centrifugal distortion constants for both torsional states are nearly equal. The following notation is employed. For example:

$$
A=\frac{A_{0}+A_{1}}{2}, \quad \Delta A=\frac{A_{0}-A_{1}}{2} .
$$

When performing a centrifugal distortion analysis according to Watson up to sixth order, we have 15 independent rotational and centrifugal distortion constants for each torsional state, or, with the just given notation, 15 sums and 15 differences of them. In addition we must fit $\Delta E_{01}$ and $F_{01}$. Because we could not fit 32 parameters to 68 transitions independently and simultaneously, some fixing of common rotational

[1] P. H. Turner, J. Chem. Soc. Faraday Trans. II 75, 317 (1979).

[2] K. Endo, T. Koshiba, H. Saito, and Y. Kamura, Nippon Kagaku Kaishi 1980, 1661 ,

[3] Ch. Keussen, U. Andresen, and H. Dreizler, Z. Naturforsch. 43 a, 469 (1988).

[4] G. Bestmann, H. Dreizler, H. Mäder, and U. Andresen, Z. Naturforsch. 35a, 392 (1980).

[5] G. Bestmann, H. Dreizler, E. Fliege, and W. Stahl, J. Mol. Struct. 97, 215 (1983).

[6] W. Stahl, G. Bestmann, H. Dreizler, U. Andresen, and R. Schwarz, Rev. Sci. Instrum. 56, 1759 (1985).

[7] H. Ehrlichmann, J. U. Grabow, H. Dreizler, N. Heineking, R. Schwarz, and U. Andresen, Z. Naturforsch. 44 a, 751 (1989). and centrifugal distortion constants for the two torsional states was necessary. Therefore a first fit was carried out with all differences of constants fixed to zero. We then tried to improve the reproduction of the measured spectrum with different combinations of differences allowed to be nonzero and included in the fit. The best reproduction was achieved with inclusion of $\Delta B_{v}, \Delta C_{v}$, and $\Delta\left(\Delta_{K}\right)_{v}$. The results are summarized in Table 2. One can see from this Table that the energy difference $\Delta E_{01}$ is only $24.8(8) \mathrm{kHz}$. The high resolution of MWFT spectroscopy was necessary to determine such a small effect with sufficient accuracy.

\section{Acknowledgement}

We thank the members of our group for help and discussions and P. Cox for sending the original version of the program MALON. Funds of the Deutsche Forschungsgemeinschaft, Fonds der Chemie, and the Land Schleswig-Holstein are gratefully acknowledged.

[8] Ch. Keussen, N. Heineking, and H. Dreizler, Z. Naturforsch. $44 \mathrm{a}, 215$ (1989).

[9] I. Merke and H. Dreizler, Z. Naturforsch. 43a, 196 (1988).

[10] J. Haekel and H. Mäder, Z. Naturforsch. 43a, 203 (1988).

[11] H. M. Pickett, J. Chem. Phys. 56, 1715 (1972).

[12] W. Gordy and R. L. Cook, Microwave Molecular Spectra, 3rd ed., John Wiley \& Sons, New York 1984, p. 331.

[13] Ch. Keussen, Dissertation, Kiel 1991, Chapt. II, p. $48-76$. 